\title{
Two dimensional modelling approach to transport properties of the TEXTOR-DED laminar zone
}

\author{
T. Eich, D. Reiser K.H. Finken \\ Institut für Plasmaphysik, Forschungszentrum Jülich GmbH, \\ Euratom Association, \\ Trilateral Euregio Cluster, \\ Jülich, Germany
}

\begin{abstract}
An important operational range for TEXTOR-DED is the case of a high ergodization at the plasma edge. Under these conditions, the edge magnetic field forms a proper ergodic layer and a laminar zone. The laminar zone is established by magnetic field lines which intersect wall elements after only a few toroidal turns (open ergodic system). For this, both the heat and particle transport in the plasma edge are dominated by the laminar zone which shows a well defined structure compared with that of the ergodic layer. For the present analysis the regions containing field lines with connection lengths corresponding to one or to two poloidal turns are of particular interest; the radial width of these regions is of the same order as the width of the unperturbed SOL of a 'classical' limiter or divertor configuration. Field lines running poloidally twice around the torus introduce a particular new feature, they impose a connection between radially separated areas and thus enhance the radial temperature and particle transport. A new 2-D modelling approach code for the laminar zone of an ergodic divertor has been developed which is strongly oriented on the topology of the magnetic field lines in the plasma edge. A 2-D finite element method is used in order to model the perpendicular transport of particles and energy. For obtaining a solution of the parallel transport, an analytical model is discussed providing expressions for averaged particle and energy sources. By considering the topological properties of the magnetic field and corresponding assumptions in the code, the transport calculation in the perturbed plasma edge gives a first insight into the generic transport properties of the TEXTOR-DED laminar zone. As an important application for later experiments the power fluxes to the wall elements are estimated. They show a strong variation perpendicular to the perturbation coils and a medium variation over the helical divertor footprints.
\end{abstract}

\section{Introduction}

The installation of the Dynamic Ergodic Divertor (DED) for TEXTOR-94 is in preparation. The scientific goal of the DED perturbation coil device in static and (quasi-static) low frequency operation is distribution of the heat fluxes from the core plasma to the wall elements over a large area, suppressing zones of high impact. Experimental results for the Tore Supra Ergodic Divertor (ED) [1-3] have shown that the local heat and particle fluxes are defined both by the near field of the coil device and by the ergodic layer. Recent numerical studies on the perturbation field generated by the DED coil device confirmed these results for TEXTOR-DED [4]. The different magnetic regions established by the DED arrangement are sketched in Fig. 1. The undisturbed inner zone of confinement is surrounded by the ergodic zone, which itself is surrounded by the so-called laminar zone. This laminar zone is characterized by relatively short connection lengths of the magnetic field lines between two intersections with the divertor target plate. In this sense the structure is quite similar to the normal SOL in a poloidal divertor tokamak.

Different modelling concepts are developed to calculate the plasma properties and heat fluxes for such (partly) ergodic 3-D magnetic field structures. For Tore Supra ED a statistical treatment has been developed for the laminar zone calculating the power deposition via the balance of the radial field line penetration into the plasma and the connection length of the field lines $[5,6]$.

For TEXTOR-DED the development of the Monte Carlo code E3-D is in progress in order to calculate the plasma properties in the real 3-D structure and include also the ergodic regions [7]. In addition to the latter mentioned progress a first analytical and numerical investigation of the field line structure has been performed for TEXTOR-DED. The results of these studies showed that, despite strong ergodization at the magnetic edge, structures with the sizes 


\section{T. Eich et al.}

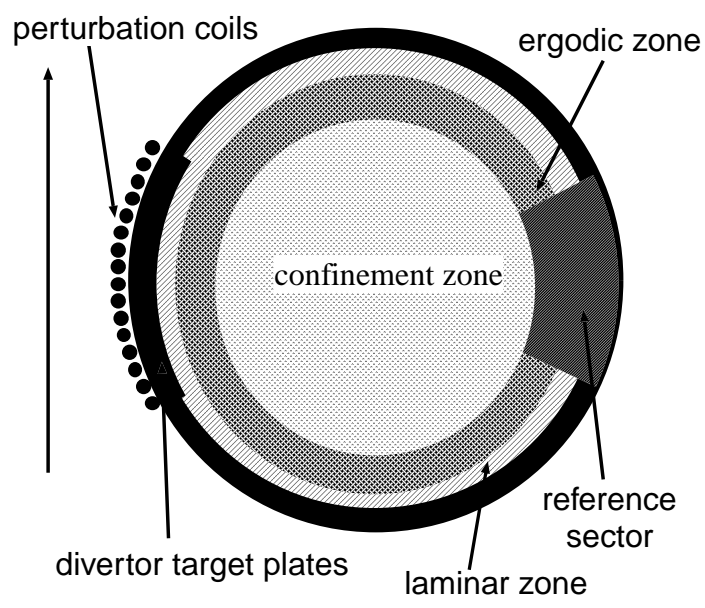

Figure 1. Sketch of the different magnetic regions generated by the superposition of the tokamak equilibrium and DED perturbation field presented in a poloidal cross section. The whole field line topology can be shown in a representative poloidal reference sector.

of typical plasma decay lengths remain as the laminar zone; this gave a clue for the first 1-D plasma modelling $[8,9]$.

The DED system allows operation under many different discharge scenarios [10]. To consider the plasma properties imposed by the magnetic field in an optimal way, the field line structure in the laminar zone is investigated in detail at first; this leads to a specific 2-D plasma modelling. The two dimensions are described by the radial and poloidal coordinates. The third dimension, i.e. transport along the magnetic field, is treated by an analytical model which is an extension of an isothermal model of the energy flux along the field lines. The assumptions used in the model restrict the applications to certain values of all possible operational discharge parameters, namely to those with a well developed laminar zone. Although the range of validity is restricted, the important case of a strong ergodization at the magnetic edge by shifting the $q=3$ magnetic surface radially close to the perturbation coil system is included, giving the model a high level of relevance for later experiments.

The main emphasis of this article is the combination of the topological studies with the calculation of plasma properties and power fluxes to the target plates. The goal of this approach is an understanding of the basic transport phenomena in relation to the topological characteristics in the laminar zone arising from the interaction of the transport along and perpendicular to the field lines. This interaction

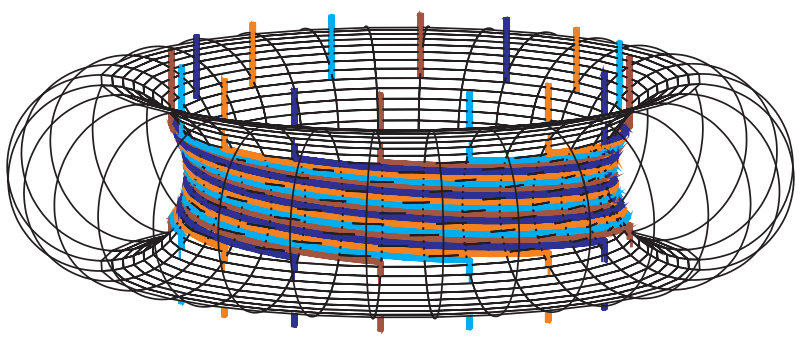

Figure 2. Sketch of the DED coils: 16 perturbation coils located on the high field side of the torus follow the field lines of the $q=3$ magnetic surface for one toroidal turn.

Table 1. Current distribution for 8 of the 16 perturbation coils

(The current distribution imposes a sinusoidal magnetic perturbation field in the radial direction.)

\begin{tabular}{lrrrrrrrrr}
\hline Coil & 1 & 2 & 3 & 4 & 5 & 6 & 7 & 8 & $\ldots$ \\
$I(\mathrm{kA})$ & 15 & 0 & -15 & 0 & 15 & 0 & -15 & 0 & $\ldots$ \\
\hline
\end{tabular}

is introduced by the radial and poloidal deflection of the field lines and was not included in the earlier 1-D model.

\section{Experimental set-up}

Figure 2 shows the schematic set-up of the perturbation coils for the DED. Sixteen perturbation coils are installed inside the vessel at the high field side (HFS). Each of the 16 coils is aligned to the field lines of the $q=3$ magnetic surface $\left(\beta_{p o l}=1\right)$ for one toroidal turn. The entrance and exit locations of each coil are in the same poloidal cut. The coils can be supplied by either DC or AC up to $10 \mathrm{kHz}$. The phasing between the currents in the coils is $90^{\circ}$, which approximates rather well a sinusoidal current distribution (see Table 1) forming a base $m=12$, $n=4$ mode, resonant to the magnetic field at the $q=3$ surface.

The maximum perturbation current for each coil is $15 \mathrm{kA}$, leading to an ergodization level characterized by a Chirikov parameter of up to 4 [11]. The coils are protected by graphite tiles forming the divertor target plates (DTPs) and facing the plasma. The real coil configuration differs from the most symmetric one by the selection of the coil feeders. The coils are installed as four bundles starting at four equidistant toroidal positions. This choice requires two additional compensation coils to maintain plasma 


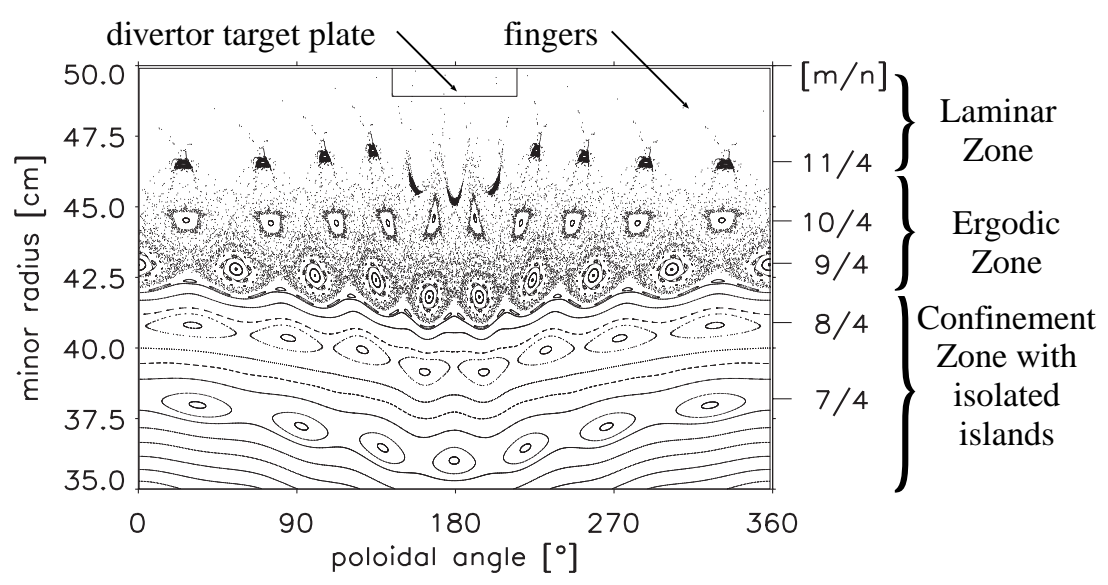

Figure 3. Poincaré plot of the edge magnetic field lines at full perturbation current.

equilibrium. A detailed report on this device is given in Ref. [12].

\section{Topological considerations}

\subsection{Poincaré plot}

The non-linear development of chaotic systems is often represented by so-called Poincaré plots, which reduce the dimension of the graph by one. For the case of the magnetic field line structure, such a plot is obtained from the sequence of intersection points of one or more selected magnetic field lines with a poloidal cut of the torus [13]. A Poincaré plot resulting from the superposition of the DED vacuum perturbation field and the equilibrium field is given in Fig. 3. It shows an example of a rather strong ergodization of the edge magnetic fields. In Fig. 3 the abscissa is the poloidal angle in the range from 0 to $360^{\circ}$ and the ordinate is the minor radius; the plasma interior (bottom part of the figure) is cut away and the DED coils are represented by the square at the top of the graph. The low field side (LFS) is on the right and left of the figure while the HFS is located at the centre. The characteristic bending of the flux surfaces and island chains results from a Shafranov shift [14]. The ergodization strength depends, for example, on $\beta_{p o l}$ and the radial location of the resonance magnetic surface; here the operating parameters for the equilibrium field are $\beta_{p o l}=0.0$ and $I_{\text {plasma }}=550 \mathrm{kA}$ with a full perturbation current of $I_{\text {pert }}=15 \mathrm{kA}$ applied. The position of the resonant $q=3$ surface is defined by the centre at $R_{0}(q=3)=174.6 \mathrm{~cm}$ and the minor radius of $r(q=3)=47.9 \mathrm{~cm}$.
The plot confirms the existence of the three different regions generated by a divertor with ergodic properties. The inner region is the confinement region with a small perturbation of the flux surfaces and some small non-overlapping island chains. Despite the strong ergodization at the boundary, the island chain on the $q=2$ surface $(m / n=8 / 4)$ is non-overlapping — probably a pre-requirement to avoid $q$-limit disruptions. The ergodic region shows a high level of stochasticity with remnants of the island chains.

In Fig. 3 these island chains are indicated by their mode number $m / n$ on the right hand side of the graph. The thin structures between the white regions are called fingers [15]. These fingers contain the field lines which connect the ergodic sea with the target plates. The finger structure will be re-identified by investigating the laminar zone. The laminar zone appears as the white region close to the wall elements; the white region does not give any detailed information because it is filled with field lines of short connection length. One aim of plotting the intersection points in a Poincaré plot is to obtain information on the plasma flow, which is directed predominantly along the field lines. Since this flow is neutralized after hitting a wall element, the field line tracing is stopped there.

\subsection{The laminar plot}

In Fig. 3, the laminar zone is practically empty of points because these field lines are quickly 'lost'; the 'loss' technique has been used in the field line tracing after an intersection of the magnetic field 


\section{T. Eich et al.}

line with the wall in order to simulate particle neutralization at the walls. Therefore a complementary imaging technique (referred to as a laminar plot) has been developed to visualize the main properties of the laminar zone. In a similar way to the Poincaré plot, a 2-D representation is chosen. This reference plane is also a poloidal cut, but because of the high poloidal mode number, only a limited poloidal section needs to be shown. Due to the $m=12$ symmetry of the coil design, the topology can be represented by a poloidal sector which covers about a twelfth of the poloidal circumference as indicated in Fig. 1. As reference area we choose one close to the outer equatorial midplane, which is either near the stagnation point of the plasma flow or is at least an area of high symmetry. Since the field lines intersect wall elements after a relatively short distance, the relevant quantity is the connection length. A laminar plot is shown in Fig. 4 for the same conditions as those in the Poincaré plot of Fig. 3. The poloidal range is not exactly $30^{\circ}$ as one might expect from the cylindrical approximation, because due to toroidal effects the pitch of the field lines is lowest at the HFS and highest at the LFS (depending on $\beta_{\text {pol }}$ ). For the case considered, this results in a span of the mode of $52.6^{\circ}$ at the LFS. A dense set of field lines is started on the poloidal reference sector and traced for 12 toroidal turns in the clockwise and counterclockwise directions around the torus using a field line tracing code $[16,17]$. Field lines intersecting an inner wall element during the 12 turns in each direction are represented by colours describing the connection length $L_{c}$ of the field line between two intersections with the wall. In many works on SOLs the distance between the intersection positions is counted as two connection lengths. This choice reflects the symmetry of the SOL for a limiter or poloidal experiment; this symmetry is not satisfied for our case and we denote by $L_{c}$ the total distance.

The whole structure of the laminar zone is shown by giving each position of the grid the colour corresponding to the calculated connection length. So the laminar plot implies the basic information about the laminar zone.

\subsection{Results of the laminar plot}

The laminar zone is composed of areas of different colours representing different field line connection lengths. The 'blue area' in the laminar plot contains field lines with connection length corresponding to one poloidal turn, i.e. three toroidal

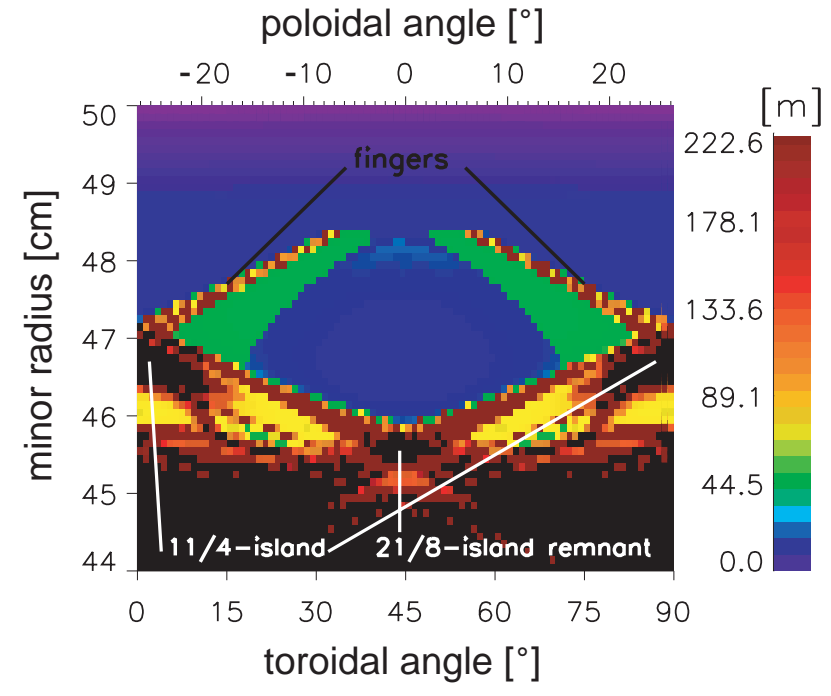

Figure 4. This laminar plot characterizes the topology of the magnetic field in the poloidal reference sector by colouring the plane corresponding to the connection lengths of the field lines.

turns $\left(L_{c} \approx 2 \pi q R\right)$, the 'green area' those with two poloidal turns $\left(L_{c} \approx 2 \times 2 \pi q R\right)$ and the 'yellow area' those with three poloidal turns $\left(L_{c} \approx 3 \times 2 \pi q R\right)$. The 'black area' contains field lines not intersecting material objects within the given tracing distance and the 'brown area' those field lines which do intersect a wall element in only one tracing direction within the $2 \times 12$ turns. The areas containing field lines with well defined $L_{c}$ are similar to those establishing the SOL of a limiter or divertor configuration.

\subsubsection{Areas with simple properties}

Although the topology seems to be rather complex, the set of field lines deflected to the wall after one poloidal turn fills a simple and continuous area and has the same properties as 'classical' configurations. Neighbouring field lines stay neighbouring and the connection lengths remain almost equal in a certain area. An important fact is that the maximum radial width of these areas is at least of the same order as typical plasma decay widths, namely $0.5-1.5 \mathrm{~cm}$. The preselected poloidal sector cuts all field lines with a given $L_{c}$ corresponding to one poloidal turn in two parts of almost equal length. For this reason the poloidal sector can be identified as a (generalized) symmetry plane known from SOL models. 
Article: Transport properties of TEXTOR-DED laminar zone

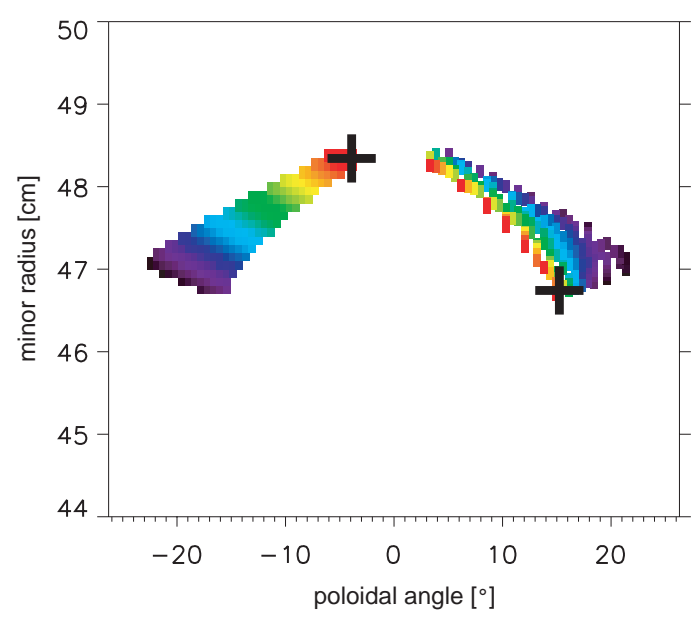

Figure 5. Radial and poloidal deflections of the magnetic field structure shown for field lines with $L_{c}$ corresponding to two poloidal turns. In this case, a given colour indicates a given field line intersecting each side of the structure. This gives an impression of deformation of the magnetic field lines due to the perturbation field. The '+' signs denote maximum radial deflection.

\subsubsection{Areas with complex properties}

The shape of the area with $L_{c}$ corresponding to two poloidal turns is somewhat more complex. Due to the fact that the field lines intersect a wall element after two poloidal turns, they cross the poloidal reference sector a second time. The deflection of these field lines can be shown by starting the field lines and marking the position with a dot in a certain colour. The intersecting position with the reference sector after one further poloidal turn is marked with a dot in the same colour. Unlike the previous figures the colours indicate the $(r, \theta)$ position in the poloidal cut. This is illustrated in Fig. 5. It can be seen that for example the field line with starting co-ordinates $\left(\theta=14.3^{\circ}, r=46.8 \mathrm{~cm}\right)$ intersects the reference sector at the position $\left(\theta=-4.2^{\circ}, r=48.3 \mathrm{~cm}\right) \mathrm{a}$ second time. This means that the radial field line deflection amounts to about $1.5 \mathrm{~cm}$. The intersections of a magnetic field line with a rather strong deflection are indicated by the symbol ' + '. Other examples would show less or even no radial (but still poloidal) deflection. The radial deflection and its importance for plasma transport is discussed in Section 5.1. The area with $L_{c}$ corresponding to three poloidal turns has similar features as the area with $L_{c}$ corresponding to two poloidal turns.

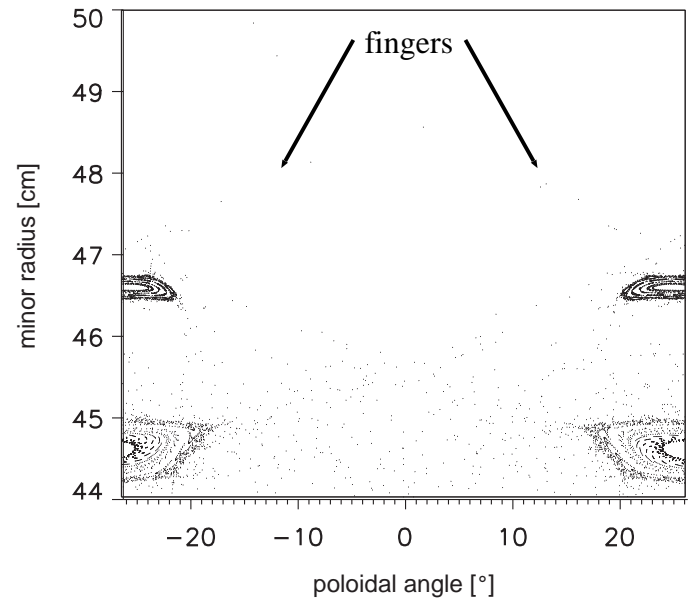

Figure 6. Zoom view of the Poincaré plot into the poloidal reference sector.

\subsubsection{Areas with ergodic properties}

The major part of the ergodic field lines, i.e. field lines with a connection length of $L_{c}>24$ toroidal turns, faces the laminar area towards the core plasma. However, a minor area with ergodic properties also intermixes with non-ergodic magnetic field lines. Nevertheless the area of ergodic field lines is also continuous and is located between the simple area and the complex area. These field lines form the very thin 'finger-like' structures. Such structures appeared already in the Poincaré plot of Fig. 3 as the thin structures close to the wall elements. Figure 6 shows a zoom of the Poincaré plot of Fig. 3 in the same frame as shown for the laminar plot. The field lines in the finger structure have long connection lengths compared with those in the neighbouring laminar area. Particles following these ergodic field lines enter the zone with short connection lengths by diffusion and then are guided quickly to the wall. For this reason the transport to the wall elements is dominated by the region of connection lengths with one or two poloidal turns.

The magnetic islands can also be recovered. The $11 / 4$ island chain is visible at the position $r=$ $46.7 \mathrm{~cm}, \theta_{1}=-26.3^{\circ}$ or $\theta_{2}=26.3^{\circ}$. The remnant of the $21 / 8$ island chain at the position $r=45.8 \mathrm{~cm}$, $\theta=0.0^{\circ}$ is visible in Fig. 4 but does not appear in the zoom view. This indicates that the island chain almost melts into the ergodic sea and appears as a 'sticky region'. 


\section{T. Eich et al.}

\section{Strategy for modelling of the laminar zone}

The aim of the previous sections was to show that the topology of the magnetic field lines can be surprisingly simple despite strong edge ergodization. Magnetic field lines with similar connection lengths $L_{c}$ form continuous clusters predominantly corresponding to one or two poloidal turns. This relatively simple structure inspired us to employ an edge modelling very similar to that of a conventional SOL. It is not our intention to include all plasma effects which are treated in sophisticated SOL codes but we are rather interested in finding the generic properties of the laminar zone. Therefore several simplifications will be used. One of those simplifications is the neglect of reionization of recycling particles (the low recycling approximation). Effects due to the curvature of the field lines will be neglected and the transport will be split into motions along the magnetic field lines and perpendicular to these. We approximate the magnetic field line bundles by a multiflux tube model where each flux tube has a length corresponding to one or two poloidal turns.

For the sake of simplicity, we assume that the particle and energy source distributions for each flux tube are uniform for one poloidal turn, an assumption that divides a certain field line in a natural way into different cells. Therefore flux tubes of one poloidal turn include one cell, while those of two poloidal turns are divided into two interconnected cells. In addition we employ (in the treatment of the parallel particle and momentum balance) the assumption that the temperature along a single field line is almost constant, which allows an analytical description of parallel transport. Both assumptions are certainly good approximations for the real scenario.

For a major review article describing the efforts on SOL modelling for limiter or divertor configurations see Ref. [18]. Even for a non-uniform distribution of the connection length of field lines in the SOL, models have been developed that treat parallel transport in the SOL as sinks for transport [19-21]. In our model, the focus of the work is kept on the non-uniform connection length and moreover on the generic new property of the laminar zone, namely the radial and poloidal deflections of the field lines. Specifically the transport on field lines corresponding to a connection length of one and two poloidal turns is kept; the topology of the field lines is treated in Sections 3.3.1 and 3.3.2.
Since the plasma profiles in the laminar zone show a dependence not only on the radial co-ordinate but also on the poloidal co-ordinate, we have to apply a 2-D approach for perpendicular transport. To solve the 2-D perpendicular transport equations, we use the commercially available partial differential equation solver PDE2-D [22], which is based on a finite element method (FEM). The parallel fluxes along field lines enter the differential equations for perpendicular transport as sources and sinks. The complete 3 -D transport problem will be treated by an operator splitting method, i.e. switching between the parallel and the perpendicular transport at each step iteratively until convergence.

In the following sections we first elucidate the relevant differential equations and their splitting into parts for parallel and perpendicular transport. We then discuss parallel transport within flux tubes of one and two poloidal turns. Finally details of the perpendicular transport and its coupling to parallel motion are discussed.

\subsection{Model equations}

In this article, the plasma will be described by a simple single fluid MHD model in a Cartesian frame, neglecting the effects due to curvature of the field lines. The basic equations used here for the stationary state are the continuity equation (1), the equation for the parallel component of the momentum (2) and the energy equation (4). For the calculation of convective transport parallel to the magnetic field a single temperature for ions and electrons, constant along one field line, is assumed. But for very strong conductive heat transport, the smooth variation of the temperature along a field line is taken into account. The perpendicular transport is assumed to be anomalous and is described by an anomalous diffusion coefficient $D_{\perp}$ and an anomalous heat conductivity $\chi_{\perp}$. The co-ordinates $x$ and $y$ refer to a plane perpendicular to the magnetic field lines and $z$ denotes the co-ordinate along the field lines. Due to the neglect of recombination and reionization effects, no volume sources for particles, momentum and energy appear in the model equations, which are

$$
\begin{gathered}
\frac{\partial}{\partial x}\left(n v_{x}\right)+\frac{\partial}{\partial y}\left(n v_{y}\right)=-\frac{\partial}{\partial z}\left(n v_{\|}\right) \\
\frac{\partial}{\partial x}\left(m n v_{x} v_{\|}\right)+\frac{\partial}{\partial y}\left(m n v_{y} v_{\|}\right) \\
=-\frac{\partial}{\partial z}\left(m n v_{\|}^{2}+2 n T\right)
\end{gathered}
$$




$$
\begin{aligned}
v_{x} & =-D_{\perp} \frac{\partial \ln n}{\partial x}, \quad v_{y}=-D_{\perp} \frac{\partial \ln n}{\partial y} \\
\frac{\partial}{\partial x} & \left(5 n v_{x} T-n \chi_{\perp} \frac{\partial T}{\partial x}\right)+\frac{\partial}{\partial y}\left(5 n v_{y} T-n \chi_{\perp} \frac{\partial T}{\partial y}\right) \\
& =-\frac{\partial}{\partial z}\left(5 n v_{\|} T+\kappa \frac{\partial T}{\partial z}\right) .
\end{aligned}
$$

Here $m$ denotes the mass of the hydrogen ion. As mentioned earlier the numerical solution of these equations for the laminar zone is performed by separation of the different transport mechanisms. First the RHS of each equation is estimated and regarded as a source for particles, momentum and energy for 2 -D perpendicular transport. The new 2 -D profiles of $n, v_{\|}$and $T$ serve as an input for calculation of the profiles along field lines. This is done by determination of appropriate source terms as described in detail in Section 4.2, which follows. These source terms then serve as new 'parallel transport sources' for perpendicular transport, and this iterative procedure is repeated until convergence.

\subsection{Parallel transport}

For cases without recycling at the edges, the heat exchange along magnetic field lines is so efficient that the plasma can be regarded as practically isothermal. This assumption allows an analytical solution of the MHD equations (1) and (2) for the transport along flux tubes, provided that the LHS, i.e. the perpendicular transport characteristics, and the temperature $T$ are known. The problem then reduces to the solution of the following equations:

$\frac{\partial}{\partial z}\left(n v_{\|}\right)=S(z)$

$\frac{\partial}{\partial z}\left(m n v_{\|}^{2}+2 n T\right)=P(z)$.

This set of equations is considered for a single field line in the range $-L_{c} / 2 \leq z \leq+L_{c} / 2$ and is accompanied by the Bohm condition for parallel velocity

$\left.v_{\|}\right|_{z=-L_{c} / 2}=-c_{s},\left.\quad v_{\|}\right|_{z=+L_{c} / 2}=c_{s}$

with sound velocity $c_{s}=\sqrt{2 T / m}$. Supposing that the source terms $S$ and $P$ are given, these equations can be integrated immediately with respect to the parallel co-ordinate $z$, leading to the equations

$\hat{n} \hat{v}_{\|}=F-1$

$\hat{n}\left(\hat{v}_{\|}^{2}+1\right)=G+2$ where $\hat{n}$ and $\hat{v}_{\|}$are the normalized density and velocity $\left(n_{*}\right.$ is the density at $\left.z=-L_{c} / 2\right)$,

$\hat{n}(z)=\frac{n(z)}{n_{*}}, \quad \hat{v}_{\|}(z)=\frac{v_{\|}(z)}{c_{s}}$

and $F$ and $G$ are the dimensionless functions

$$
\begin{aligned}
& F(z)=\frac{1}{n_{*} c_{s}} \int_{-L_{c} / 2}^{z} S\left(z^{\prime}\right) d z^{\prime} \\
& G(z)=\frac{1}{m n_{*} c_{s}^{2}} \int_{-L_{c} / 2}^{z} P\left(z^{\prime}\right) d z^{\prime} .
\end{aligned}
$$

Substituting $\hat{n}$ from Eq. (8) into Eq. (9) leads to a quadratic equation for $\hat{v}_{\|}$with the physical solution $\left(\hat{v}_{\|} \rightarrow 0\right.$ in the limit $\left.F \rightarrow 1\right)$

$\hat{v}_{\|}=\frac{G / 2+1-\sqrt{(G / 2+1)^{2}-(F-1)^{2}}}{F-1}$

and it follows for $\hat{n}$ that

$\hat{n}=\frac{(F-1)^{2}}{G / 2+1-\sqrt{(G / 2+1)^{2}-(F-1)^{2}}}$.

Equations (13) and (14) represent the unique solution of Eqs (5) and (6) for given source functions $S(z)$ and $P(z)$.

The unknown value of $n_{*}$ can be determined by the Bohm condition in the form

$\left.F\right|_{z=+L_{c} / 2}=2+\left.\frac{1}{2} G\right|_{z=+L_{c} / 2}$

or equivalently

$n_{*}=\frac{1}{4 m c_{s}^{2}} \int_{-L_{c} / 2}^{L_{c} / 2}\left[2 m c_{s} S\left(z^{\prime}\right)-P\left(z^{\prime}\right)\right] d z^{\prime}$.

These solutions will be employed in the simulation in the following way: we determine profiles of the density in the perpendicular plane to obtain its values at one or more points along the particular field lines. The formulas above will then be used to calculate appropriate source terms $S$ and $P$ which are assumed to be constant along a single poloidal turn for each field line (Section 4.3). These source profiles will be used as corrected sources in a subsequent 2D calculation providing new profiles for density and temperature.

\subsection{Reconstruction of source profiles}

In this section we describe our method of reconstructing the source profiles $S(z)$ and $P(z)$ from given values for the density and/or parallel velocity in the discrete cells along the magnetic field lines. The momentum transfer $P(z)$ will be in most cases 


\section{T. Eich et al.}

neglected such that the solution will be similar to the SOL flow discussed in the cited works [18-21]. Only if the source term $S(z)$ is negative does one have to keep the momentum transfer for this cell because otherwise the solution of the flow would become complex. At present, a more accurate 3-D modelling is in preparation giving the chance to model also the momentum transfer for any case. However, the 2D approach has the advantage that it needs only a small amount of computation time for a spatially dense grid. For this purpose it is necessary to invert Eqs (13) and (14), which is possible for always positive source functions $S(z)$, so that $F(z)$ is monotonically increasing, and one obtains

$F=1+\frac{2(G / 2+1) \hat{v}_{\|}}{1+\hat{v}_{\|}^{2}}$
$F=1 \pm \sqrt{2(G / 2+1) \hat{n}-\hat{n}^{2}}$.

The sign in Eq. (18) depends on whether $z \leq z_{0}$ (negative sign for the cell with $\hat{v}_{\|} \leq 0$ ) or $z>z_{0}$ (positive sign for the cell with $\hat{v}_{\|}>0$ ), where $z_{0}$ is the position of the stagnation point with $\hat{v}_{\|}=0$. For the assumed piecewise constant profile of $S$ mentioned above, and when using the assumption $G(z)=0$ (i.e $P(z)=0$ ), one needs $N$ equations like (17) or (18) for a field line with $N$ poloidal turns, i.e. $N$ values $n_{1}, n_{2}, \ldots, n_{N}$ must be prescribed. It should be emphasized that with the simplification $P(z)=0$ the solution of Eqs (17) or (18) together with Eq. (15) will provide us with values $S_{1}, S_{2}, \ldots, S_{N}$ only if the values $n_{i}$ obey certain constraints which reflect the conservation laws (5) and (6). So, for example, in the case of two poloidal turns the densities $n_{1}$ and $n_{2}$ cannot differ by more than a factor of 2 . To remove this restriction in the simulation procedure, we will introduce a momentum source $P$ proportional to $S$ whenever the density profiles given by the $2-\mathrm{D}$ calculation do not fit into the scheme with $P(z)=0$. In the other cases we will neglect momentum sources in the parallel momentum balance equation. This concept will be illustrated now for the important cases of field lines with one and two poloidal turns, respectively.

\subsection{Examples for the parallel flow}

\subsubsection{Case 1: Field lines with one poloidal turn}

The source profile is described by a constant value $S$ for the whole field line, i.e. for $-L_{c} / 2 \leq z \leq$ $+L_{c} / 2$. It follows from Eq. (15) that

$S=\frac{2 n_{*} c_{s}}{L_{c}}$.

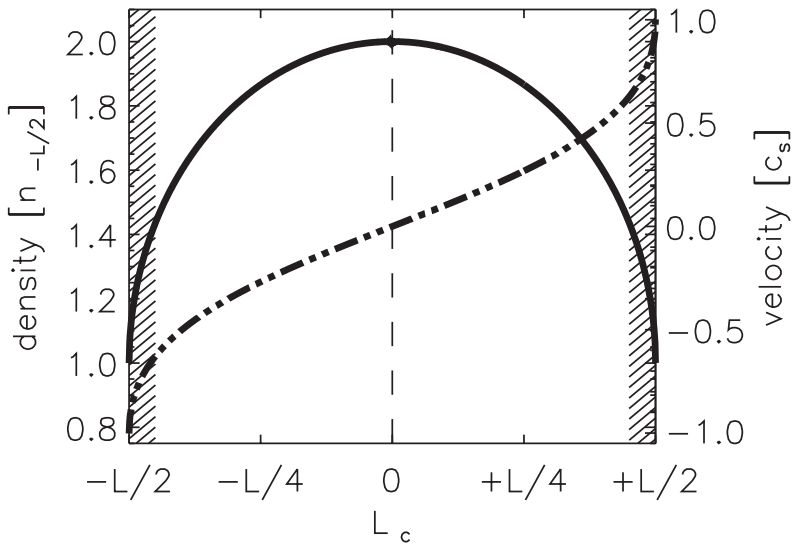

Figure 7. Profiles of $\hat{n}$ and $\hat{v}_{\|}$along a field line of one poloidal turn, with $S>0$ and $P=0$. The hatched area denote the areas of perturbation.

Prescribing now the density $n_{0}$ at the stagnation point $z_{0}=0$ at the midpoint of the cell, one obtains from Eq. (8)

$n\left(z_{0}\right)=n_{0}=2 n_{*}$

and thus

$S=\frac{n_{0} c_{s}}{L_{c}}$.

Figure 7 shows an example of a homogeneously distributed source. This case represents the simple, low recycling, SOL and has been discussed, for example, by Stangeby and McCracken [18]. The density and the flow are symmetric around the stagnation point and, towards the ends, the density decreases by a factor of 2 . In the further treatment, this example represents the case of magnetic flux tubes which intersect the walls after one poloidal turn just as in the conventional SOL.

\subsubsection{Case 2: Field lines with two poloidal turns, $S_{1}>0$ and $S_{2} \geq 0$}

The next example is representative of flux tubes which go twice poloidally around the torus before intersecting the walls. Such flows we assign two cells of equal length where each of these cells has its homogeneously distributed source, i.e. now the source profile is assumed of the form

$S(z)=\left\{\begin{array}{lll}S_{1} & \text { for } & -L_{c} / 2 \leq z \leq 0 \\ S_{2} & \text { for } & 0 \leq z \leq+L_{c} / 2\end{array}\right.$

with positive constants $S_{1}$ and $S_{2}$. Referring again to Eq. (15) it follows that

$S_{1}+S_{2}=\frac{4 n_{*} c_{s}}{L_{c}}$. 
Prescribing the densities at the midpoints of both cells by $n_{1}=n\left(-L_{c} / 4\right)$ and $n_{2}=n\left(L_{c} / 4\right)$ one obtains from Eq. (18)

$1-\sqrt{2 \hat{n}_{1}-\hat{n}_{1}^{2}}=\frac{S_{1} L_{c}}{4 n_{*} c_{s}}$

$1+\sqrt{2 \hat{n}_{2}-\hat{n}_{2}^{2}}=\frac{S_{1} L_{c}}{2 n_{*} c_{s}}+\frac{S_{2} L_{c}}{4 n_{*} c_{s}}$

where $\hat{n}_{i}$ is defined by

$\hat{n}_{i}=\frac{n_{i}}{n_{*}}$.

Inserting Eq. (23) into Eq. (25) and subtracting Eq. (24) results in

$\sqrt{2 n_{1} n_{*}-n_{1}^{2}}+\sqrt{2 n_{2} n_{*}-n_{2}^{2}}=n_{*}$.

This equation can be solved numerically to obtain $n_{*}$. The formulas for the source terms can also be written in the following form, which is very convenient for the following discussion of the heat source terms:

$S_{i}=\frac{n_{i} c_{s}}{L_{c} / 2}\left(\frac{2}{\hat{n}_{i}}-2 \sqrt{\frac{2}{\hat{n}_{i}}-1}\right)$

where $i=1,2$.

Obviously a solution of Eq. (27) is possible only in the range (cf. Eq. (14))

$\frac{1}{2} \max \left(n_{1}, n_{2}\right) \leq n_{*} \leq \min \left(n_{1}, n_{2}\right)$

and consequently the ratio $\max \left(n_{1}, n_{2}\right) / \min \left(n_{1}, n_{2}\right)$ cannot exceed a value of 2 in the case with $P=0$ and $S>0$. This can cause problems in the iteration procedure, because it is not guaranteed that the solution of the 2-D procedure fulfils this constraint. In such cases a momentum source $P \neq 0$ will be used as explained in Section 4.4.3.

Figure 8 shows the density profile for a case in which the ratio of the sources is assumed to be $5: 1$. As expected, the flow pattern is no longer symmetric and the profile is not differentiable at the midpoint due to the discontinuous change in the sources. In addition, the stagnation point moves into the direction of the stronger source. Nevertheless, some flow characteristics remain unchanged: the densities at the right and left hand free ends of the flux tube are equal to half of the density at the stagnation point and the flow losses are equal for both ends.

Figure 9 shows a case where the source of the right hand cell is zero. Under this condition, the flow in the left hand cell is similar to that shown in Fig. 7. The flow leaving the right hand end of cell 1 passes at the speed of sound through cell 2 and is lost there. Again the density and the loss rate at the ends of the flux tube are equal.

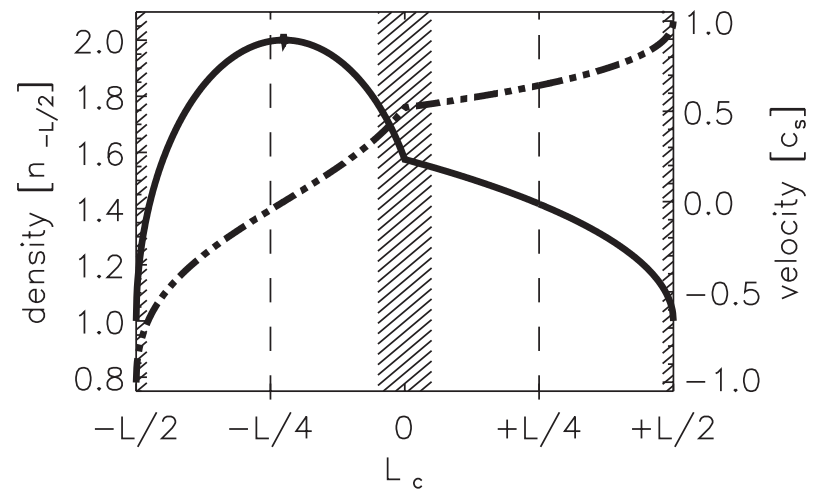

Figure 8. Profiles of $\hat{n}$ and $\hat{v}_{\|}$along a field line of two poloidal turns, with $S_{1}, S_{2}>0$ and $P=0$.

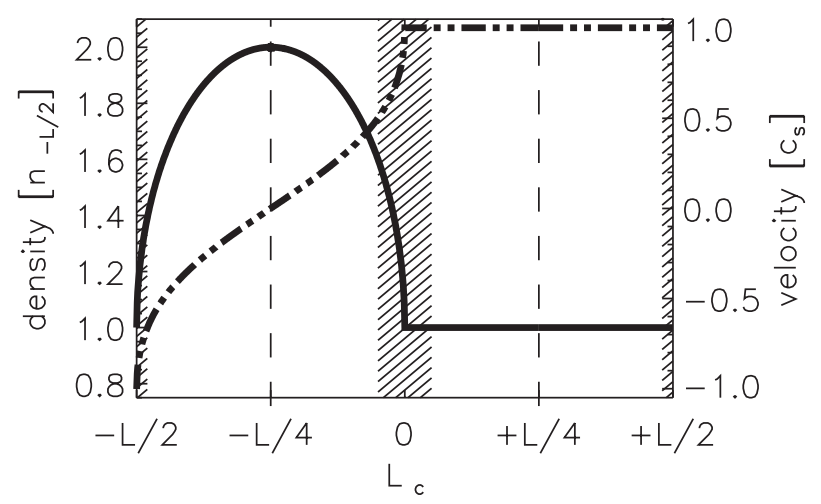

Figure 9. Profiles of $\hat{n}$ and $\hat{v}_{\|}$along a field line of two poloidal turns, with $S_{1}>0, S_{2}=0$ and $P=0$.

4.4.3. Case 3: Field lines with two poloidal turns, $S_{1} / S_{2}<0$

Again the source profile is assumed to be of the form (22), but now we allow either $S_{1}$ or $S_{2}$ to be negative. Because of the negative source term it is possible to obtain density profiles where the ratio $\max \left(n_{1}, n_{2}\right) / \min \left(n_{1}, n_{2}\right)$ of the densities at the midpoints of the two cells can take any value. But if we would still neglect a momentum source, i.e. set $P=0$, Eqs (13) and (14) would not have any (real) solution. Instead of calculating appropriate source terms $P$ in a way similar to that presented for $S$, we simplify matters by requiring that the flow velocity $v_{\|}$in the cell with $S<0$ is constant and equal to $\pm c_{s}$, consistent with the boundary conditions. This seems to be reasonable, because like the wall (where the Bohm criterion is stated) the cell with $S<0$ works as a perfect sink. By this we avoid the solution of complicated non-linear equations to determine $P$ and replace assumptions about the momentum source profile by a linear dependence $P \sim S$. 


\section{T. Eich et al.}

The expressions for $P$ are derived in the following way. Assuming $v_{\|}=$const Eqs (5) and (6) are written as

$v_{\|} \frac{\partial n}{\partial z}=S(z)$

$m v_{\|}^{2} \frac{\partial n}{\partial z}+2 T \frac{\partial n}{\partial z}=P(z)$.

Inserting Eq. (30) into (31) leads to

$P(z)=\left(m v_{\|}+m \frac{c_{s}^{2}}{v_{\|}}\right) S(z)$.

The value of the flow velocity is fixed by the boundary conditions in the respective cell. For the boundary conditions (7) this means that for the case with $S_{1}<0, S_{2}>0$,

$P(z)=\left\{\begin{array}{ccc}-2 m c_{s} S_{1} & \text { for } & -L_{c} / 2 \leq z \leq 0 \\ 0 & \text { for } & 0 \leq z \leq+L_{c} / 2\end{array}\right.$

and for $S_{1}>0, S_{2}<0$,

$P(z)=\left\{\begin{array}{ccc}0 & \text { for } & -L_{c} / 2 \leq z \leq 0 \\ +2 m c_{s} S_{2} & \text { for } & 0 \leq z \leq+L_{c} / 2 .\end{array}\right.$

The reconstruction formulas are particularly simple for these cases. For $n_{1}<n_{2}$ (i.e. $S_{1}<0$ and $S_{2}>0$ ) one has to use

$n_{*}=2 n_{1}-\frac{1}{2} n_{2}$

$S_{1}=\frac{4 n_{1} c_{s}}{L_{c}}-\frac{2 n_{2} c_{s}}{L_{c}}, \quad S_{2}=\frac{2 n_{2} c_{s}}{L_{c}}$

and for the case $n_{1}>n_{2}$ (i.e. $S_{1}>0$ and $S_{2}<0$ )

$n_{*}=\frac{1}{2} n_{1}$

$S_{1}=\frac{2 n_{1} c_{s}}{L_{c}}, \quad S_{2}=\frac{4 n_{2} c_{s}}{L_{c}}-\frac{2 n_{1} c_{s}}{L_{c}}$.

Obviously these formulas allow a unique solution for the source profiles to be obtained for any ratio $\max \left(n_{1}, n_{2}\right) / \min \left(n_{1}, n_{2}\right)>2$. In the limits $n_{1}=2 n_{2}$ and $n_{1}=n_{2} / 2$ the results of Eqs (35) and (36), respectively, agree with those of Eqs (27) and (28), as should be the case.

Figure 10 shows an example with a negative source in the right hand cell. The characteristic features are the linear decay of the density in the cell with negative source term $S$ and the fact that the densities at the ends are no longer equal.

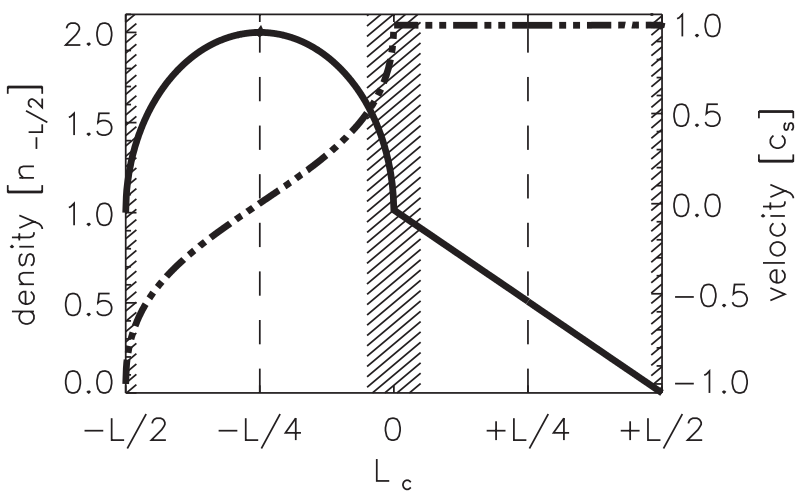

Figure 10. Profiles of $\hat{n}$ (solid line) and $\hat{v}_{\|}$(chain line) along a field line of two poloidal turns, with $S_{1}>0$, $S_{2}<0, P_{1}=0$ and $P_{2}=2 m c_{s} S_{2}$.

\subsection{Heat source terms}

In this section the 'parallel heat source' terms of Eq. (4), i.e. its RHS, are estimated. For convective heat transport we use the particle source terms derived in the last sections and additionally a two point estimation for conductive heat transport. For heat transport to the walls a sheath transmission factor $\gamma=6.5$ is introduced to take into account the effects of sheath potential [18]. First we rewrite the RHS of Eq. (4) as

$$
\begin{aligned}
Q(z) & =-\frac{\partial}{\partial z}\left(5 n v_{\|} T+\kappa \frac{\partial T}{\partial z}\right) \\
& \approx-\frac{\partial}{\partial z}\left(5 n v_{\|}\right) T-\frac{\partial}{\partial z}\left(\kappa \frac{\partial T}{\partial z}\right)
\end{aligned}
$$

with electron heat conductivity

$\kappa=2.07 \times 10^{22} T^{5 / 2}\left(\mathrm{eV}^{5 / 2} \mathrm{~ms}\right)^{-1}$.

For field lines with two poloidal turns the temperature $T$ is estimated as

$T=\frac{n_{1} T_{1}+n_{2} T_{2}}{n_{1}+n_{2}}$

with $T_{i}$ the temperature in cell $i$ of a particular field line, and the gradient $\partial T / \partial z$ is calculated by use of

$\frac{\partial T}{\partial z} \approx \frac{T_{2}-T_{1}}{L_{c} / 2}$.

The first term in Eq. (37), the convective heat transport term, is expressed by particle source terms $S(z)$ of the form we presented in the last sections, but with a modification to take into account the effect of the sheath potential on fluxes to the walls. For field lines 
with one and two poloidal turns the heat sources due to convection can be written as

$Q^{\text {conv }}=\frac{\left|\Delta q^{\text {conv }}\right|}{L_{c} / 2}$

with $\Delta q^{\text {conv }}$ the difference between the convective heat fluxes entering and leaving a certain cell along the field line. So for a field line with one poloidal turn $\Delta q^{c o n v}$ is the difference between the two fluxes to the walls, and for field lines with two poloidal turns $\Delta q^{c o n v}$ is the difference between a flux to the wall and a flux through the interface of the two cells.

\subsubsection{Case 1: Field lines with one poloidal turn}

For field lines with connection lengths corresponding to one poloidal turn the flux tube has only one cell intersecting the wall on both ends. With the sheath conditions for heat transport and the assumption of a constant profile the heat source term is described by

$Q^{c o n v}=\gamma \frac{n_{0} c_{s}}{L_{c}} T$.

4.5.2. Case 2: Field lines with two poloidal turns, $S_{1}>0$ and $S_{2} \geq 0$

For field lines with connection lengths corresponding to two poloidal turns the flux tubes are divided into two cells. Each cell ends on one side at the wall and is connected to the other cell of the flux tube at the other end,

$Q_{i}^{\text {conv }}=\frac{n_{i} c_{s}}{L_{c} / 2}\left[\frac{\gamma}{\hat{n}_{i}}+5\left(\frac{1}{\hat{n}_{i}}-2 \sqrt{\frac{2}{\hat{n}_{i}}-1}\right)\right] T$

where $i=1,2$.

4.5.3. Case 3: Field lines with two poloidal turns, $S_{1} / S_{2}<0$

For this case we have again to distinguish two cases depending on in which cell a negative particle source is assumed.

For $S_{1}<0, S_{2}>0$ one obtains

$Q_{1}^{c o n v}=\frac{n_{1} c_{s}}{L_{c} / 2}\left[\gamma\left(2-\frac{1}{2} \frac{n_{2}}{n_{1}}\right)-\frac{5}{2} \frac{n_{2}}{n_{1}}\right] T$

$Q_{2}^{\text {conv }}=\frac{n_{2} c_{s}}{L_{c} / 2}\left(\frac{\gamma}{2}+\frac{5}{2}\right) T$

and for $S_{1}>0$ and $S_{2}<0$

$Q_{1}^{c o n v}=\frac{n_{1} c_{s}}{L_{c} / 2}\left(\frac{\gamma}{2}+\frac{5}{2}\right) T$

$Q_{2}^{\text {conv }}=\frac{n_{2} c_{s}}{L_{c} / 2}\left[\gamma\left(2-\frac{1}{2} \frac{n_{1}}{n_{2}}\right)-\frac{5}{2} \frac{n_{1}}{n_{2}}\right] T$.
(2)

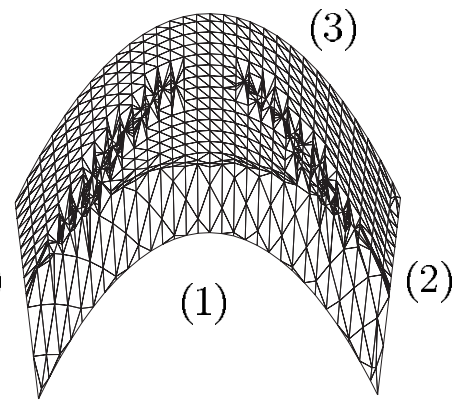

Figure 11. Calculation grid, which is framed by four boundaries corresponding to (1) confinement area, (2) periodic boundary condition and (3) wall. The ergodic, complex and simple areas are easily discernible and are denoted by decreasing density of triangles.

\subsection{2-D modelling of cross-field transport}

\subsubsection{Calculational domain}

The perpendicular transport calculation is based on an FEM. This technique requires a triangulation of the area in which the differential equation is solved. In our calculations we are solving transport equations in the whole area shown in Fig. 11. The calculational grid contains the ergodic area (I) (all field lines with a connection length of more than two times poloidally around the torus are counted as ergodic), the complex area (II) and the simple area (III). The transport problem in the ergodic area (I) is treated in a simplified manner. The effect of ergodicity is taken into account by enhancing the transport coefficients as is discussed, for example, in Refs $[11,2]$. The distorted field lines are therefore not traced to calculate the parallel transport in the ergodic region as well. (Even though the method can be extended to field lines with a connection length of several $q \cdot 2 \pi r$.)

Different methods for automatic triangulation are offered as mathematical packages. Nevertheless we have chosen a self-written routine forcing the triangles to cover areas of field lines belonging to the ergodic or the laminar areas, respectively. Each vortex $j$ of a triangle represents the intersection of a single field line with the computational domain and therefore the information on parallel transport for each triangle is known in detail. Field lines in the complex area intersect the grid and are represented by two vortices corresponding to the two cells denoted by the index $i=1,2$. For the computation a linear ansatz function is chosen which 


\section{T. Eich et al.}

ensures that the FEM solver uses the three vortices of the triangles at which the solution of the parallel transport is given. Any other choice of ansatz function (quadratic, cubic or quartic) has to use more points inside the triangle $(6,10$ or 15$)$ and would force an interpolation of the data from the parallel transport solution. Such an interpolation would use more data points for the calculation but only the same amount of physical information about parallel transport as for the linear ansatz.

\subsubsection{The PDE2D solver}

The type of equations we are going to solve is

$\frac{\partial}{\partial x}\left(D_{\perp} \frac{\partial n}{\partial x}\right)+\frac{\partial}{\partial y}\left(D_{\perp} \frac{\partial n}{\partial y}\right)=S(x, y)$

$$
\begin{aligned}
\frac{\partial}{\partial x} & \left(5 D_{\perp} \frac{\partial n}{\partial x} T+n \chi_{\perp} \frac{\partial T}{\partial x}\right) \\
& +\frac{\partial}{\partial y}\left(5 D_{\perp} \frac{\partial n}{\partial y} T+n \chi_{\perp} \frac{\partial T}{\partial y}\right)=Q(x, y) .
\end{aligned}
$$

The different areas I-III in the calculational domain are described by different values for the particle and energy diffusion coefficient and expressions for $S(x, y)$ and $Q(x, y)$ as derived in Sections 4.3-4.5. For simplicity the diffusive transport in the ergodic area is assumed to be isotropic. An anisotropy due to convective transport along the field lines does not appear in this model, because the $x-y$ plane is by definition perpendicular to the magnetic field lines. The indices 'new' and 'old' denote the values of the last iteration step and the actual values, respectively. For each flux tube $j$ and cell $i$ the following parameters are used:

Area I (ergodic area):

$D_{\perp}=1.0 \mathrm{~m}^{2} / \mathrm{s}, \quad \chi_{\perp}=3.0 \mathrm{~m}^{2} / \mathrm{s}$

$S_{j i}^{\text {new }}(x, y)=0$

$Q_{j i}^{\text {new }}(x, y)=0$.

Area II and area III (laminar area):

$D_{\perp}=0.5 \mathrm{~m}^{2} / \mathrm{s}, \quad \chi_{\perp}=1.5 \mathrm{~m}^{2} / \mathrm{s}$

$S_{j i}^{\text {new }}(x, y)=\frac{n_{j i}^{\text {new }}}{n_{j i}^{\text {old }}} E_{j i} S_{j i}^{\text {old }}(x, y)$

$Q_{j i}^{\text {new }}(x, y)=\frac{n_{j i}^{\text {new }} T_{j i}^{\text {new }}}{n_{j i}^{\text {old }} T_{j i}^{\text {old }}} E_{j i} Q_{j i}^{\text {old }}(x, y)$.
In Eqs (52) and (53) the sources $S_{j i}^{\text {old }}(x, y)$ and $Q_{j i}^{\text {old }}(x, y)$ are computed according to our reconstruction recipe but are modified by a density correction factor $E_{j i}$ and are linearized with respect to the density $n_{j i}^{\text {new }}$ to improve the convergence of the iterative procedure. The density correction factor $E_{j i}$ takes into account the fact that the diffusion process between two neighbouring cells in different flux tubes depends on the density in the complete cell and not alone on the values at the midpoints of the cells, which are used to reconstruct the parallel transport source terms. The density correction factor is defined as

$E_{j i}=\frac{1}{L_{c} / 2} \int_{(\Delta L)_{j i}} \frac{n(z)}{n_{j i}} d z$

where $(\Delta L)_{j i}$ is the length of the cell $i$ corresponding to vortex $j$. For cells with one poloidal turn one has always $E_{j i}=1 / 2+\pi / 8$, and for cells with $S \leq 0$ the density correction factor is simply equal to 1 . In the other cases its value is in the range $1 / 2+\pi / 8 \leq E_{j i} \leq$ 1 and can be computed by elementary integration.

\subsubsection{Boundary conditions}

The boundary condition for perpendicular transport is imposed at the different arcs shown in Fig. 11. For the interface between the ergodic area and the core plasma at arc (1) values for the density and temperature are prescribed as

$n_{(1)}=1 \times 10^{19} \mathrm{~m}^{-3}$ and $T_{(1)}=50 \mathrm{eV}$.

For arc (2) the fluxes across the boundaries are assumed to vanish. This is certainly a good approximation due to the slight variation of the temperature and density along the field lines and the much larger extension and symmetry of the topology in the poloidal direction,

$\left(n v_{x} a_{x}+n v_{y} a_{y}\right)_{(2)}=0$

and

$\left(-n \chi_{\perp} \frac{\partial T}{\partial x} a_{x}-n \chi_{\perp} \frac{\partial T}{\partial y} a_{y}\right)_{(2)}=0$.

For arc (3) the boundary conditions are imposed by particle and heat fluxes corresponding to a certain decay length, i.e. for particle transport

$\left(n v_{x} a_{x}+n v_{y} a_{y}\right)_{(3)}=-D_{\perp} \frac{n}{\lambda_{n}}$

and for heat transport

$\left(-n \chi_{\perp} \frac{\partial T}{\partial x} a_{x}-n \chi_{\perp} \frac{\partial T}{\partial y} a_{y}\right)_{(3)}=-n \chi_{\perp} \frac{T}{\lambda_{T}}$ 
where $a_{x}$ and $a_{y}$ are the components of the unit vector on the corresponding surface. The decay lengths are chosen as $\lambda_{n}=0.015 \mathrm{~m}$ and $\lambda_{T}=0.02 \mathrm{~m}$.

\subsection{Summary of the iterative procedure}

The iterative algorithm for our 2-D modelling (Fig. 12) can be summarized as follows:

0. First we start with guessed profiles for the 2-D distribution of density and temperature in the calculational domain.

1. For the given 2-D profiles, source terms as defined in Sections 4.3-4.5 are computed.

2. By using the analytical profiles corresponding to the values of $S_{j i}^{\text {old }}$ and $Q_{j i}^{\text {old }}$ the density correction $E_{j i}$ is computed and the modified sources $S_{j i}^{\text {new }}$ and $Q_{j i}^{n e w}$ are determined.

3. The sources $S_{j i}^{\text {new }}$ and $Q_{j i}^{\text {new }}$ are used for the 2-D calculation and the continuity and heat balance equations are solved.

4. The density and temperature profiles are actualized and are used in the next iteration step starting again at point 1 .

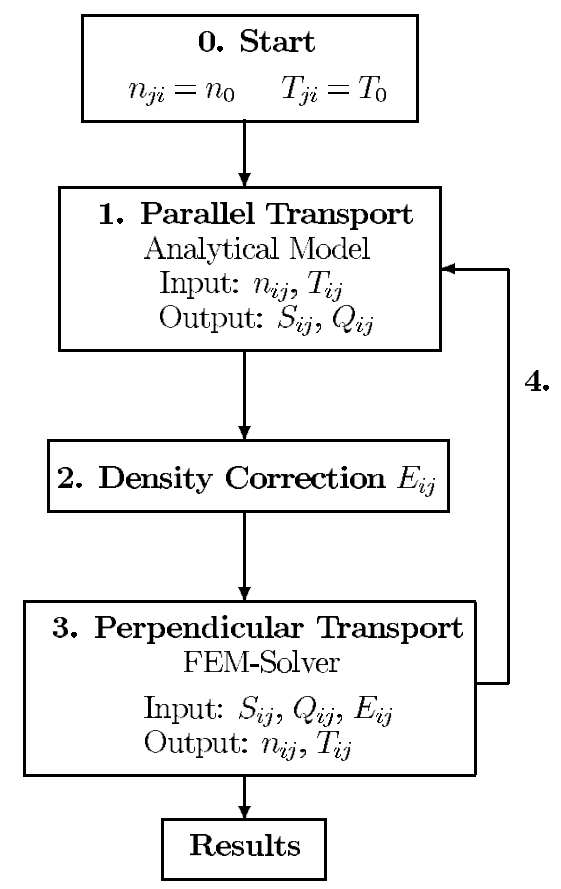

Figure 12. Schematic view of the iterative procedure.

\section{Results}

\subsection{Density and temperature distribution}

Results for the application of our model to the DED are shown in Figs 13-16. In Figs 13 and 14 the density and temperature profiles, respectively, are given. For this calculation, the temperature and density at the arc (1) facing the ergodic plasma (Fig. 11) are prescribed to be $n_{(1)}=1 \times 10^{19} \mathrm{~m}^{-3}$ and $T_{(1)}=50 \mathrm{eV}$. The density distributions are clearly influenced by the different connection lengths in the

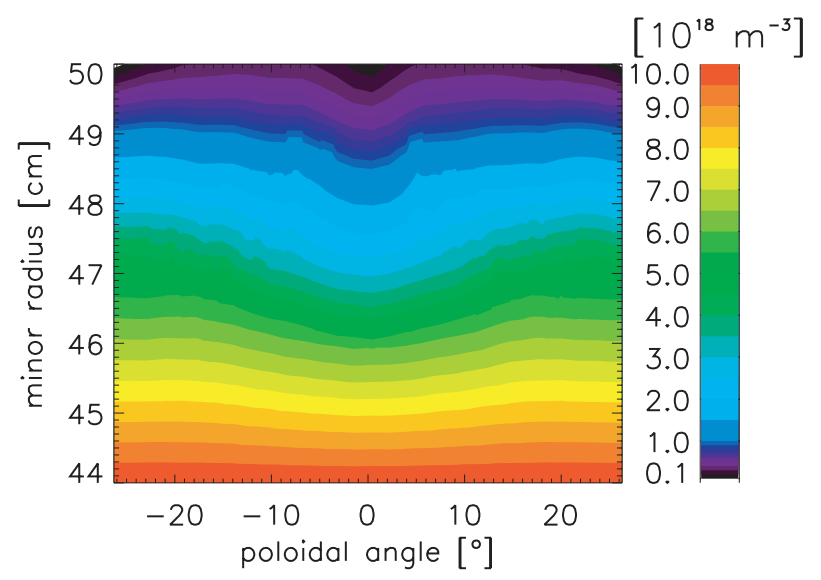

Figure 13. Calculated density distribution in the laminar zone in units of $10^{18} \mathrm{~m}^{-3}$. The density decays on curved arcs mostly aligned to the boundary between the laminar and ergodic zones. The effects of additional radial and poloidal particle fluxes are small.
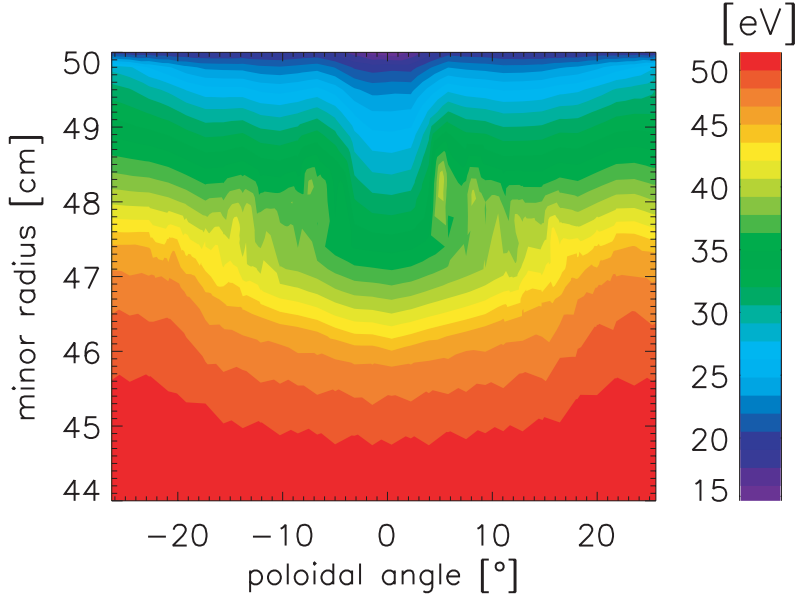

Figure 14. Calculated temperature distribution in units of electronvolts. The field line structure causes a strong deformation due to the additional radial and poloidal energy fluxes. 


\section{T. Eich et al.}

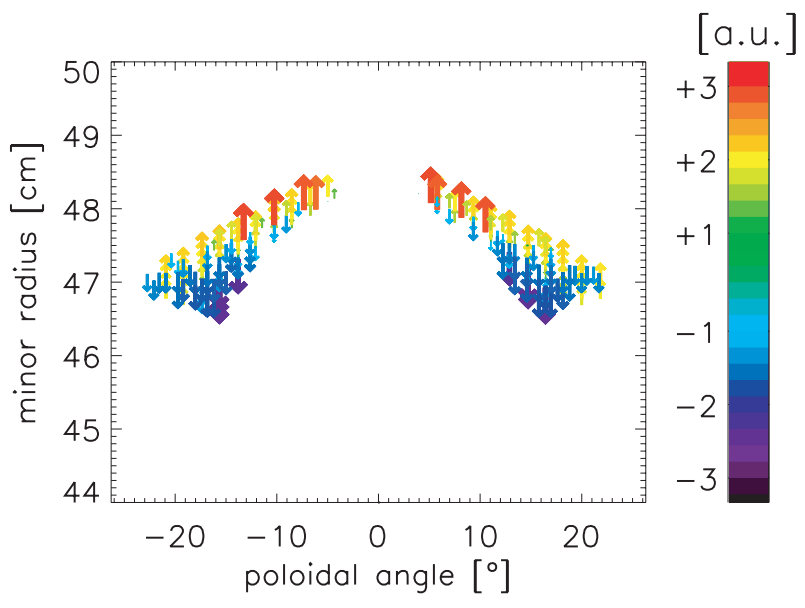

Figure 15. Calculated energy fluxes arising due to the radial and poloidal deflections of the field lines. Energy and particles are transported from the area with downward directed arrows to the area with upward directed arrows.

poloidal and radial directions. Due to our choice for the diffusion coefficient the plasma profiles decay on a characteristic length close to the experimentally observed decay length.

The influence of the field structure is much more obvious for the temperature distribution. In Fig. 15 the additional fluxes caused by the radial convection and conduction terms are illustrated. A comparison with Fig. 5 demonstrates the effects of perturbed field structure. The field lines with a connection length corresponding to two poloidal turns connect two positions in the laminar zone. The enhancement of radial transport in the laminar zone arises from convective and conductive parallel particle and energy fluxes. The different values of density and temperature at the two positions lead to the exchange fluxes discussed earlier and cause a deformation of the plasma distribution in the laminar zone. The much stronger deformation of the temperature distribution compared with the density distribution is in good qualitative agreement with probe measurements at the Tore Supra ED [23]. The maximum variation of the temperature along a field line amounts to about $10 \%( \pm 5 \mathrm{eV})$, in agreement with our previous assumption of a small temperature variation.

\subsection{Power load on the wall elements}

The calculated power load to the wall is shown in Fig. 16. Here the horizontal axis represents the toroidal angle from 0 to $360^{\circ}$ and the vertical co- ordinate the poloidal angle, again from 0 to $360^{\circ}$ (the HFS of the torus is located at $180^{\circ}$ ). The flux density under shallow incidence with $\alpha$ as the impinging angle is given by

$q_{\|, \text {wall }}=\gamma \Gamma_{\|, \text {wall }} T \sin \alpha$.

To achieve more data points the irregular grid is transformed onto a uniform rectangular grid as introduced in Section 3. Thus the number of data points for the power load distribution is increased by a factor of 10 . The data on the uniform grid are computed by an interpolation of the values on the grid points. The integrated value of the heat flux over the inner wall, i.e. the total power $P_{\text {wall }}$ deposited by convection, is found to be $607.4 \mathrm{~kW}$ for this given case. This corresponds to a typical TEXTOR-94 discharge in RI mode [24] with a heating power of $2.5 \mathrm{MW}$ and a radiation level of $\approx 71.7 \%$. The power deposition is calculated for the intersecting structure of magnetic field lines introduced in earlier works [9].

It should be recalled that the intersecting structure includes no field lines which enter the volume only at the HFS and can be identified with a kind of private flux region for the DED coil system. Because the connection length of such field lines in the private flux region is very short $(\leq 7 \mathrm{~m})$, the amount of deposited power will be small. Moreover, a treatment of the region is only possible taking into account the complete ergodic geometry of the magnetic edge, which cannot be covered by the model presented here.

An important result for later diagnostics is the variation of power deposition perpendicular to and along the helical footprints on the DTPs. The maximum local power load in the calculation is $367.4 \mathrm{~kW} \mathrm{~m}^{-2}$; this high value may appear to be in contradiction with a power load balanced distribution by field line ergodization. Since these high heat flux zones are already known from Tore Supra ED operation [1-3], the dynamic option of TEXTORDED has been introduced. By this field rotation, the divertor strike zones (helical foot prints) are smeared over the whole surface of the divertor target plate, i.e. over several square metres. First experiments implementing a dynamic magnetic perturbation are performed by CSTN-4 resonant magnetic perturbation experiments $[25,26]$. A comparison of the power load of the model presented here and the results of our earlier 1-D model shows some differences. Taking the same values in the 1-D model [9] for the density and temperature in the ergodic area the convective power load amounts to $1208.3 \mathrm{~kW}$ with maximum values of 

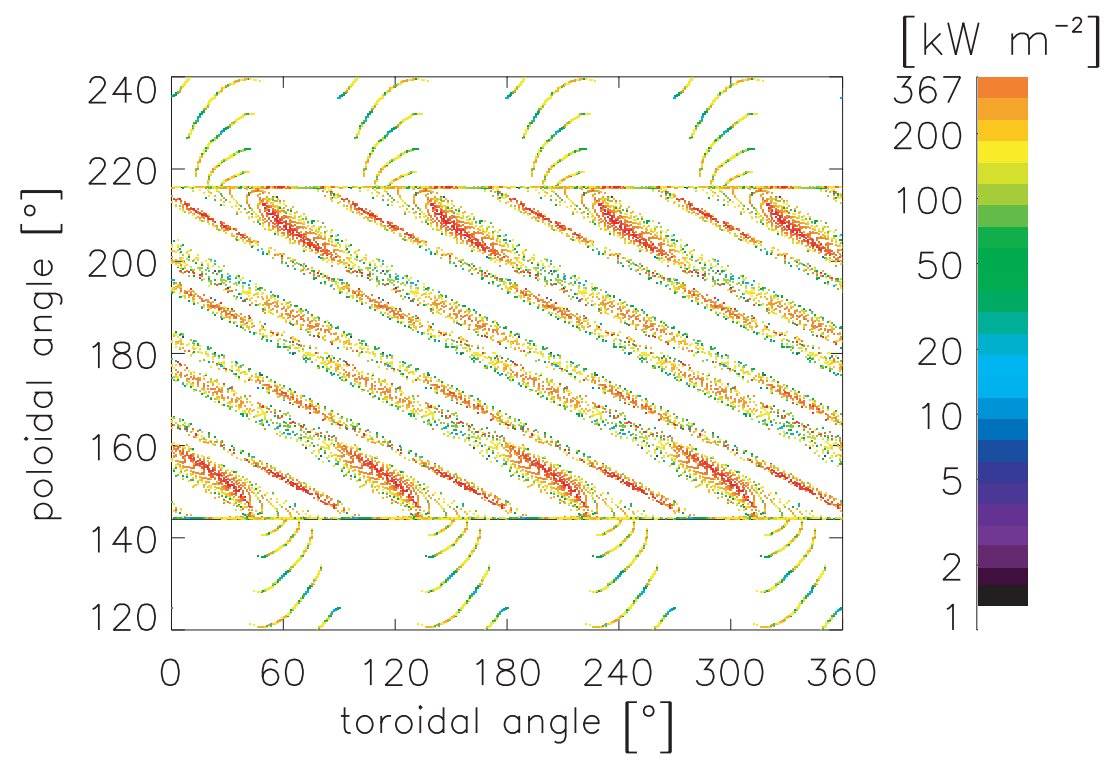

Figure 16. Calculated power load onto the TEXTOR-DED inner wall elements. The power deposition pattern shows a strong variation along and perpendicular to the helical co-ordinate.

$575 \mathrm{~kW} \mathrm{~m}^{-2}$ for the local power flux density. However, the resulting pattern of both models shows similar properties as, for example, the area of the maximum power flux density. This result is certainly due to the strong orientation on the magnetic field structure.

\section{Summary}

In this article the basic properties of the TEXTOR-DED laminar zone are studied. A laminar plot is used as an imaging technique to visualize the topology of the laminar zone. For cases of strong ergodization, the laminar zone is very prominent. This gives us the chance to discover the effects of these properties on the particle and energy transport with a simplified SOL model. The main emphasis in our model is placed on perpendicular transport; parallel transport is treated in an analytical model and may give necessary improvements. The results show that the density and temperature distributions depend on both the radial and the bi-normal co-ordinate. The effect of enhanced radial transport caused by radial deflection is explained by the model. Although the modelling is restricted to field lines of one or two poloidal turns this new feature for parallel transport along radially deflected field lines has been discovered.
As a first application the heat fluxes (without recycling and reionizing effects) have been computed indicating variations of local heat fluxes along and perpendicular to the helical intersecting structure.

An essential prerequisite of our modelling procedure is separation of the laminar and ergodic zones in the plasma edge. The ergodic area is treated rather simply, but this simplification reduces the computational effort enormously, because a detailed study of the highly complicated ergodic area requires much more theoretical and numerical work. Of course, only a comparison between numerical results and experimental data will show which level of complication of the problem is necessary and which reduction of our approach is tolerable. The feeling is that the assumptions of this article are valid if the laminar zone is well pronounced with respect to the thickness of the ergodic layer, i.e. if edge ergodization is satisfactorily high, as can indeed be achieved, as demonstrated by other modellings [27].

\section{Acknowledgements}

The authors are grateful to M. Hassler and A. Kaleck for providing detailed instructions for the magnetic field calculations. 


\section{T. Eich et al.}

\section{References}

[1] Ghendrih, P., Grosman, A., Capes, H., Plasma Phys. Control. Fusion 38 (1996) 1653.

[2] Ghendrih, P., Grosman, A., Nguyen, F., J. Nucl. Mater. 220-222 (1995) 511.

[3] Grosman, A., et al., Contrib. Plasma Phys. 38 (1998) 82.

[4] Eich, T., Finken, K.H., in Controlled Fusion and Plasma Physics (Proc. 25th Eur. Conf. Prague, 1998), Vol. 22C, European Physical Society, Geneva (1998) 1824.

[5] Ghendrih, P., Grosman, A., J. Nucl. Mater. 241243 (1997) 517.

[6] Nguyen, F., et al., Nucl. Fusion 37 (1997) 743.

[7] Runov, A., et al., in Controlled Fusion and Plasma Physics (Proc. 26th Eur. Conf. Maastricht, 1999), Vol. 23J, European Physical Society, Geneva (1999) 195.

[8] Finken, K.H., Nucl. Fusion 37 (1997) 583.

[9] Finken, K.H., Eich, T., Kaleck, A., Nucl. Fusion 38 (1998) 515.

[10] Finken, K.H., et al., Nucl. Fusion 39 (1999) 637.

[11] Abdullaev, S.S., et al., Phys. Plasmas 6 (1999) 153.

[12] Finken, K.H. (Ed.), Fusion Eng. Des. 37 (1997) No. 3.

[13] Lichtenberg, J.A., Liebermann, M.A., "Regular and stochastic motion", Applied Mathematical Science, Vol. 38, Springer-Verlag, Berlin (1983).

[14] Kaleck, A., Contrib. Plasma. Phys. 39 (1999) 367.

[15] Hassler, M., Dynamische Ergodisierung an TEXTOR-94, Konzeptanalyse und Auslegungsuntersuchung, Rep. 3616, Forschungszentrum Jülich (1998).
[16] Kisslinger, M., The Gourdon Code, Max-Planck Institut für Plasmaphysik, Garching, personal communication.

[17] Kaleck, A., Hassler, M., The Gourdon Code, specified version, Forschungszentrum Jülich (1997).

[18] Stangeby, P.C., McCracken, G.M., Nucl. Fusion 30 (1990) 1225.

[19] Lingertat, J., et al., Plasma Phys. Control. Fusion 29 (1987) 1365.

[20] Günther, K., Contrib. Plasma Phys. 28 (1988) 365.

[21] Feng, Y., et al., J. Nucl. Mater. 241-243 (1997) 930.

[22] Sewell, G., Adv. Eng. Software 17 (1993) 105.

[23] Gunn, J.P., et al., in Controlled Fusion and Plasma Physics (Proc. 25th Eur. Conf. Prague, 1998), Vol. 22C, European Physical Society, Geneva (1998) 19.

[24] Messiaen, A.M., Ongena, J., Samm, U., Phys. Rev. Lett. 77 (1996) 2487.

[25] Takamura, S., et al., in Controlled Fusion and Plasma Physics (Proc. 25th Eur. Conf. Prague, 1998), Vol. 22C, European Physical Society, Geneva (1998) 722.

[26] Kobayashi, M., et al., Nucl. Fusion 40 (2000) 181.

[27] Ghendrih, P., et al., in Controlled Fusion and Plasma Physics (Proc. 24th Eur. Conf. Berchtesgaden, 1997), Vol. 21A, Part IV, European Physical Society, Geneva (1997) 1685.

(Manuscript received 8 November 1999

Final manuscript accepted 12 July 2000)

E-mail address of T. Eich:

th.eich@fz-juelich.de

Subject classification: B0, Tt; F2, Tt 